\title{
Sustained swimming capacity of Atlantic salmon
}

\author{
Malthe Hvas ${ }^{*}$, Frode Oppedal
}

Institute of Marine Research, 5984 Matredal, Norway

\begin{abstract}
Sustained swimming in fish relies on aerobic metabolism alone and has been defined as swimming speeds that can be maintained for $200 \mathrm{~min}$. However, few studies have tested swimming performances at constant velocities for such durations. Instead, the limits of sustainable swimming are often defined indirectly as speeds where anaerobic metabolism is required. Atlantic salmon Salmo salar aquaculture is currently expanding to more exposed locations that experience occasional strong current velocities, and thus a more practical assessment of the sustained swimming capacity is needed to ensure acceptable animal welfare. To this purpose, we performed swim tunnel respirometry experiments with groups of larger post-smolts $(\sim 800 \mathrm{~g})$ at $13^{\circ} \mathrm{C}$. First, the average critical swimming speed $\left(U_{\text {crit }}\right)$ was determined $\left(97.2 \mathrm{~cm} \mathrm{~s}^{-1}\right)$; then, sustained swimming trials were conducted in which fish were forced to swim up to $4 \mathrm{~h}$ at 60,80 or $100 \%$ of the average $U_{\text {crit }}$. All fish endured 60 and $80 \% U_{\text {crit }}$ for $4 \mathrm{~h}$, while only 2 avoided fatigue at $100 \% U_{\text {crit }}\left(\mathrm{n}=24\right.$ treatment $\left.^{-1}\right)$. A decrease in oxygen consumption rate over time at the sub- $U_{\text {crit }}$ intensities indicated swimming became more efficient and aerobic. To explain variation in swimming performance within the group, $U_{\text {crit }}$ and fatigue time were correlated with relative ventricular mass, condition factor and weight. However, these parameters were poor predictors of swimming performance. In conclusion, Atlantic salmon possess the aerobic capacity for continuous high-intensity swimming of at least $80 \% U_{\text {crit }}$ for several hours. This adds important temporal insight to welfare guidelines related to current velocities in aquaculture.
\end{abstract}

KEY WORDS: Endurance swimming $\cdot U_{\text {crit }} \cdot$ Respirometry $\cdot$ Exposed aquaculture

\section{INTRODUCTION}

Sustained swimming does not result in fatigue and, in theory, can therefore be maintained indefinitely. Physiologically, this means that propulsion should be strictly aerobically fueled. In salmonids, this involves recruitment of the thin layer of slow red muscle fibres on the lateral sides, while fast white muscle fibres remain inactive (Hudson, 1973, Bone et al. 1978, Wilson \& Egginton 1994). Homeostasis is then maintained with no accumulation of lactate, depletion of glycogen stores or other respiratory or osmotic disturbances.

Sustained swimming has been defined as swimming speeds that can be maintained for $200 \mathrm{~min}$ (Beamish 1978). Although this definition is widely

${ }^{*}$ Corresponding author: malthe.hvas@imr.no cited, studies on swimming capabilities rarely test fish at constant velocities for $\geq 200 \mathrm{~min}$ (e.g. Brett 1967, Cotterell \& Wardle 2004). Instead, the most common assessment of swimming capacity is the critical swimming speed $\left(U_{\text {crit }}\right)$, where a stepwise increase in swimming speed at intervals typically varying between 15 and $60 \mathrm{~min}$ is continued until the fish fatigues (Brett 1964, Plaut 2001, Farrell 2007). Therefore, $U_{\text {crit }}$ represents the highest prolonged swimming speed a fish can maintain for a limited time (minutes) and is inherently powered by both aerobic and anaerobic metabolism at the final swimming velocities (Wilson \& Egginton 1994).

To assess the speed limit of sustainable swimming in salmonids, several studies have used standard $U_{\text {crit }}$ protocols while deploying various approaches

() The authors 2017. Open Access under Creative Commons by Attribution Licence. Use, distribution and reproduction are unrestricted. Authors and original publication must be credited. 
to reveal when the transition from strictly aerobic to a mixture of aerobic and anaerobic metabolism occurs. Methods have involved calculations of aerobic swimming efficiency and power propulsion (Webb 1971), acid-base status and change in swimming behaviour (Kiceniuk \& Jones 1977), measurements of intramuscular lactate and phosphocreatine (Burgetz et al. 1998), and electromyography to measure when large-amplitude white muscle traces are present (Sisson \& Sidell 1987, Wilson \& Egginton 1994, Beddow \& McKinley 1999). These studies found that aerobic metabolism predominates in salmonids swimming up to 70 to $90 \%$ of their $U_{\text {crit }}$ (e.g. Jones 1982, Burgetz et al. 1998, Beddow \& McKinley 1999). This represents an impressive aerobic capacity for continued high-intensity swimming in salmonids compared to other groups of fish, such as cyprinids, that only sustain 30 to $50 \%$ of their $U_{\text {crit }}$ aerobically (Jones 1982).

Interestingly, regardless of the experimental approach, these previous studies did not test whether the fish were able to maintain their highest sustainable swimming speed for $\geq 200$ min, thereby not confirming whether those derived swimming speeds actually were truly sustainable. A less theoretical and more practical assessment of sustained swimming capabilities in farmed Atlantic salmon is now needed because of the current trend of establishing salmon sea cage farms at more exposed locations (Holmer 2010, Bjelland et al. 2015). With exposed sites positioned in high-energy environments with occasional strong water currents that potentially impact fish welfare, acceptable current velocities need to be defined. In this regard, $U_{\text {crit }}$ was recently suggested as a useful welfare parameter for acceptable peak currents in sea cages (Remen et al. 2016). However, $U_{\text {crit }}$ only represents the acceptable limit during short-duration strong current events and provides little information about whether the fish will cope or reach fatigue at intermediate current velocities if exposed for several hours.

The purpose of this study was to test the sustained swimming capacity of Atlantic salmon at $U_{\text {crit }}$ and sub- $U_{\text {crit }}$ speeds for durations of up to $4 \mathrm{~h}$ within a large swim tunnel respirometer. The oxygen consumption rate $\left(\mathrm{MO}_{2}\right)$ here provides a measurement of the steady-state aerobic energy expenditure during prolonged swimming at constant velocities. A secondary objective was to assess the variation in swimming performance between individuals and whether this variation could be explained by size, condition factor $(K)$ and relative ventricular mass (RVM).

\section{MATERIALS AND METHODS}

\section{Animals}

Prior to the experiments, farmed Atlantic salmon Salmo salar (Aquagen) had been kept for 6 mo at the Institute of Marine Research, Matre, Norway, in large circular tanks ( $3 \mathrm{~m}$ diameter, $5.3 \mathrm{~m}^{3}$ volume) with an inflow of $120 \mathrm{l} \mathrm{min}^{-1}$ under a simulated natural photoperiod at low currents. Water temperature was maintained at $13^{\circ} \mathrm{C}$, dissolved oxygen was not allowed to fall below $85 \%$ saturation and salinity was $34 \%$ (full-strength seawater). Feed was given in excess daily between 10:00 and 14:00 h through automatic feeding devices (Nutra, $3 \mathrm{~mm}$ pellet size; Skretting). Experiments were performed in November and December 2016 in accordance with Norwegian laws and regulations for procedures and experiments on live animals under permit number 9776 .

\section{Experimental setup}

Swim trials were conducted in a large Brett-type swim tunnel respirometer with a swim section $248 \mathrm{~cm}$ long by $36 \mathrm{~cm}$ in diameter. The technical specifications and flow properties of this setup have previously been described (Remen et al. 2016, Bui et al. 2016, Hvas et al. 2017). To observe undisturbed fish behaviour, a camera was placed behind the rear grid of the swim section adjacent to an oxygen sensor (RINKO ARO-FT; JFE Advanced). Oxygen concentration was logged every 2 s (MiniSoft SD200W; SAIV A/S Environmental Sensors \& Systems) and was kept above $85 \%$ saturation at all times by rapid intermittent flushing with water from a large, thermally controlled reservoir connected to the swim tunnel setup, which also served to keep the temperature constant at $13 \pm 0.1^{\circ} \mathrm{C}$. The top opening at the rear end could be partially removed when fish were placed in or taken out of the swim tunnel setup.

A large swim tunnel was chosen to better accommodate the natural swimming behaviour of Atlantic salmon and to prevent underestimations of the true swimming capacity of the fish, which has been known to occur in smaller setups (Tudorache et al. 2007, Deslauriers \& Kieffer 2011, Remen et al. 2016). Groups of 8 fish were tested simultaneously per swim trial to reduce the volume-to-biomass ratio, so reliable $\mathrm{O}_{2}$ traces could be made within $10 \mathrm{~min}$. Furthermore, small, single-fish respirometers closely resemble the conditions for confinement stress trials of salmonids (Pottinger \& Carrick 1999, 
Vindas et al. 2016). Therefore, small groups of Atlantic salmon swimming together in a larger volume may reduce stress levels during experiments. Swimming in schools is also likely to reduce the cost of transport and therefore improve the swimming performance in some species (Hartwell \& Otto 1978, Herskin \& Steffensen 1998, Svendsen et al. 2003).

The fish rarely overlapped, meaning that for the most part they never exceeded $10 \%$ of the cross-sectional area of the swim tunnel. As a consequence, solid blocking effects were not corrected for (Bell \& Terhune 1970, Plaut 2001).

\section{Swim trial protocols}

Fish were gently netted and quickly moved to the swim tunnel, which was placed in the same room as the holding tanks, and were allowed to acclimate to their new surroundings at $20 \mathrm{~cm} \mathrm{~s}^{-1}$ ( 0.46 body lengths $[\mathrm{BL}] \mathrm{s}^{-1}$ ) overnight before the swim trials commenced the following day.

In the first series of experiments, the average $U_{\text {crit }}$ of the stock was defined by a stepwise increase in current velocity of $20 \mathrm{~cm} \mathrm{~s}^{-1}$ every $30 \mathrm{~min}$ until all fish reached fatigue. Fatigue was defined as when a fish was no longer able to continue swimming even when encouraged to do so by a touch to the tail. Three replicate $U_{\text {crit }}$ trials were conducted with a total of 24 fish.

In the second series of experiments, sustained swimming capabilities were assessed by forcing fish from the same stock to swim for $4 \mathrm{~h}$ at a constant water current velocity, defined as a percentage of the average $U_{\text {crit }}$ obtained in the previous experiment. A total of 3 replicate trials at 60,80 and $100 \%$ of the average group $U_{\text {crit }}$ were made. Swim speed was increased by $20 \mathrm{~cm} \mathrm{~s}^{-1}$ every 15 min until the desired velocity was reached. The time was noted when a fish fatigued and it was then quickly removed from the setup, while the fish that endured all $4 \mathrm{~h}$ were noted to have completed the swim challenge.

$\mathrm{MO}_{2}$ measurements were made during all swim trials. In the $U_{\text {crit }}$ protocol, the system was closed for $20 \mathrm{~min}$ and then flushed for $10 \mathrm{~min}$ at each increment interval, while the closed and flush periods were reduced to 10 and $5 \mathrm{~min}$ respectively during the sustained swimming protocols. Dissolved oxygen saturation was never allowed to fall below $85 \%$. To ensure this, the closed period was reduced to $8 \mathrm{~min}$ and the flushing period increased to 7 min during sustained swimming at $100 \% U_{\text {crit }}$.
After swim trials, fish were removed from the experimental setup and immediately killed with a quick blow to the head. Fish weight $(W)$ and fork length $\left(L_{\mathrm{f}}\right)$ were measured and the ventricle was dissected out and weighed.

\section{Calculations}

$U_{\text {crit }}$ was calculated according to Brett (1964):

$$
U_{\text {crit }}=U_{\mathrm{f}}+\frac{t_{\mathrm{f}} U_{\mathrm{i}}}{t_{\mathrm{i}}}
$$

where $U_{\mathrm{f}}$ is the last completed swim speed, $t_{\mathrm{f}}$ is the time where fatigue was reached at the final swim speed, $t_{\mathrm{i}}$ is the time interval at each speed, and $U_{\mathrm{i}}$ is the magnitude of the swim speed increment.

$\mathrm{MO}_{2}$ was calculated from the change in oxygen concentration over time for each of the closed periods in all the swim trials. The cost of transport (CoT) at the various swimming speeds during the $U_{\text {crit }}$ trials was calculated as $\mathrm{mg} \mathrm{O}_{2} \mathrm{~kg}^{-1} \mathrm{~km}^{-1}$. A parabola was fitted to these values to express CoT as a function of swimming speed. The minimum of this function then served as an approximation of the optimum CoT swimming speed. RVM was calculated as the percentage of the ventricular mass of the total body weight of the fish. $K$ was calculated according to Ricker (1975) as $100\left(W / L_{\mathrm{f}}^{3}\right)$.

\section{Statistics}

A 1-way ANOVA with Tukey's post hoc test was used to test for differences between groups in $W, L_{\mathrm{f}}, K$ and RVM, and a Shapiro-Wilk test was used to confirm equal variance in these data. A linear regression fit was made between swimming capacities and various parameters to explore possible correlation patterns. Pearson's correlation coefficient was used to determine the statistical significance and strength of these correlations (SigmaPlot v.12.3; Systat Software). The level of significance for all tests was set at $\alpha=0.05$.

\section{RESULTS}

The values of $W, L_{\mathrm{f}}, K$ and RVM for the different test groups are summarized in Table $1 . W$ and $L_{\mathrm{f}}$ were similar between groups. However, the $60 \% U_{\text {crit }}$ group had larger RVM and lower $K$ compared to the other groups, indicating that their bodies were slightly leaner. 
Table 1. Weight $(W)$, fork length $\left(L_{\mathrm{f}}\right)$, relative ventricular mass (RVM) and condition factor $(K)$ of the 4 test groups of Atlantic salmon $(n=24)$. Superscript letters indicate a significant difference $(\mathrm{p}<0.05)$. Data are means $\pm \mathrm{SE}$

\begin{tabular}{|lcccc|}
\hline Groups & $W(\mathrm{~g})$ & $L_{\mathrm{f}}(\mathrm{cm})$ & $\mathrm{RVM}$ & $K$ \\
\hline$U_{\text {crit }}$ test & $849 \pm 36$ & $43.0 \pm 0.6$ & $0.085 \pm 0.002^{\mathrm{a}}$ & $1.06 \pm 0.02^{\mathrm{a}}$ \\
$60 \% U_{\text {crit }}$ sustained & $777 \pm 50$ & $42.9 \pm 0.6$ & $0.097 \pm 0.003^{\mathrm{b}}$ & $0.96 \pm 0.03^{\mathrm{b}}$ \\
$80 \% U_{\text {crit }}$ sustained & $836 \pm 27$ & $42.7 \pm 0.5$ & $0.089 \pm 0.003^{\mathrm{a}}$ & $1.07 \pm 0.02^{\mathrm{a}}$ \\
$100 \% U_{\text {crit }}$ sustained & $916 \pm 40$ & $43.9 \pm 0.6$ & $0.091 \pm 0.003^{\mathrm{a}}$ & $1.07 \pm 0.02^{\mathrm{a}}$ \\
\hline
\end{tabular}

with occasional burst and glide movements and a tendency to fall back until they hit the rear grid.

$\mathrm{MO}_{2}$ during sustained swimming had distinct curve profiles at each of the intensities, averaging 509.9, 415.5 and $306.3 \mathrm{mg} \mathrm{O}_{2} \mathrm{~kg}^{-1} \mathrm{~h}^{-1}$ at 100,80 and $60 \% U_{\text {crit }}$ respectively (Fig. 2). Since most fish fatigued in the $100 \%$ group, the measured $\mathrm{MO}_{2}$ should be a good approximation of the aver-

The average $U_{\text {crit }}$ was $97.2 \pm 1.6 \mathrm{~cm} \mathrm{~s}^{-1}(2.27 \pm$ $0.04 \mathrm{BL} \mathrm{s}^{-1}, \mathrm{n}=24$ ), where all fish fatigued while swimming at either 100 or $120 \mathrm{~cm} \mathrm{~s}^{-1}$. The water current velocities for sustained swimming trials at 60,80 and $100 \%$ of the average $U_{\text {crit }}$ were therefore set to 58,78 and $97 \mathrm{~cm} \mathrm{~s}^{-1}$ respectively.

No fish fatigued while swimming at 60 and $80 \%$ $U_{\text {crit }}$ for $4 \mathrm{~h}$ (Fig. 1). Only 2 out of 24 fish endured $4 \mathrm{~h}$ at $100 \% U_{\text {crit, }}$ with the majority fatigued within the first $2 \mathrm{~h}$ (Fig. 1). $L_{\mathrm{f}}$ in the $100 \%$ group ranged from 36 to $49.5 \mathrm{~cm}$, meaning that fish were swimming at relative speeds between 2.69 and $1.96 \mathrm{BL} \mathrm{s}^{-1}$, which may have explained differences in endurance time at this absolute current speed. However, the linear regression of $\mathrm{BL} \mathrm{s}{ }^{-1}$ against fatigue time had an almost horizontal slope and an $\mathrm{R}^{2}$ of 0.006 (Fig. 1), which suggests that fatigue time was independent of the differences in relative swimming speeds of individual fish in this group. Fish approaching fatigue showed increasingly unsteady swimming behaviour

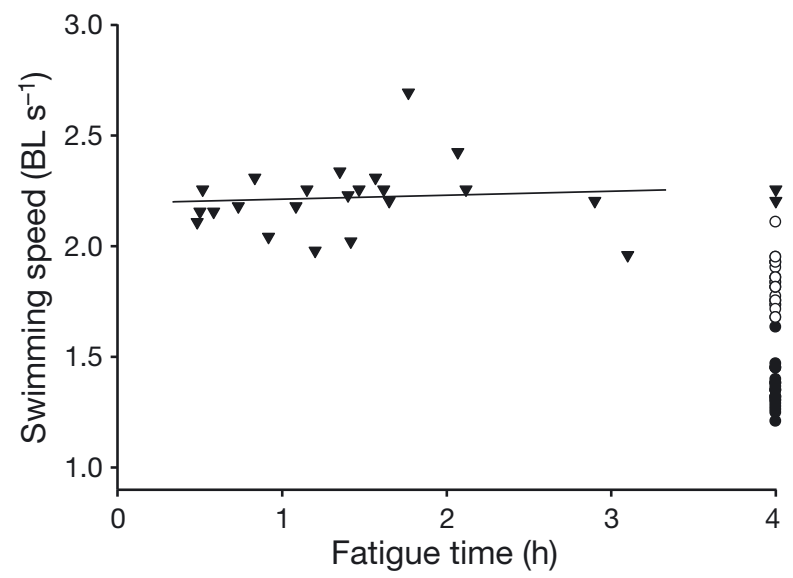

Fig. 1. Relative swimming speed (body length [BL] s ${ }^{-1}$ ) versus time to fatigue during sustained swimming trials of Atlantic salmon. Points at the $4 \mathrm{~h}$ mark represent individual fish that completed the trial. Filled circles, open circles and triangles correspond to swimming speeds of 58,78 and $97 \mathrm{~cm} \mathrm{~s}^{-1}$ respectively. Line represents a linear regression for fatigued fish $\left(y=0.017 x+2.194, R^{2}=0.006\right)$ age maximum rate of oxygen consumption $\left(\mathrm{VO}_{2 \max }\right)$. This implies that sustained $\mathrm{MO}_{2}$, expressed as a percentage of $\mathrm{VO}_{2 \max }$ in the 60 and $80 \%$ groups, on average corresponded to 60.1 and $81.4 \%$ of $\mathrm{VO}_{2 \max }$ respectively.

There was a tendency for $\mathrm{MO}_{2}$ to slightly decrease over time during sustained swimming. A linear regression of $\mathrm{MO}_{2}$ versus time produced negative slopes of -5.35 and $-7.44 \mathrm{mg} \mathrm{O} \mathrm{kg}^{-1} \mathrm{~h}^{-1}$, with corresponding highly significant Pearson correlation factors of -0.77 and -0.74 respectively $(p<0.001)$. The pattern in $\mathrm{MO}_{2}$ at $100 \%$ was more ambiguous since fish had to be removed from the setup between most measurement periods, and since too few fish eventually remained to obtain a proper oxygen trace in the latter periods.

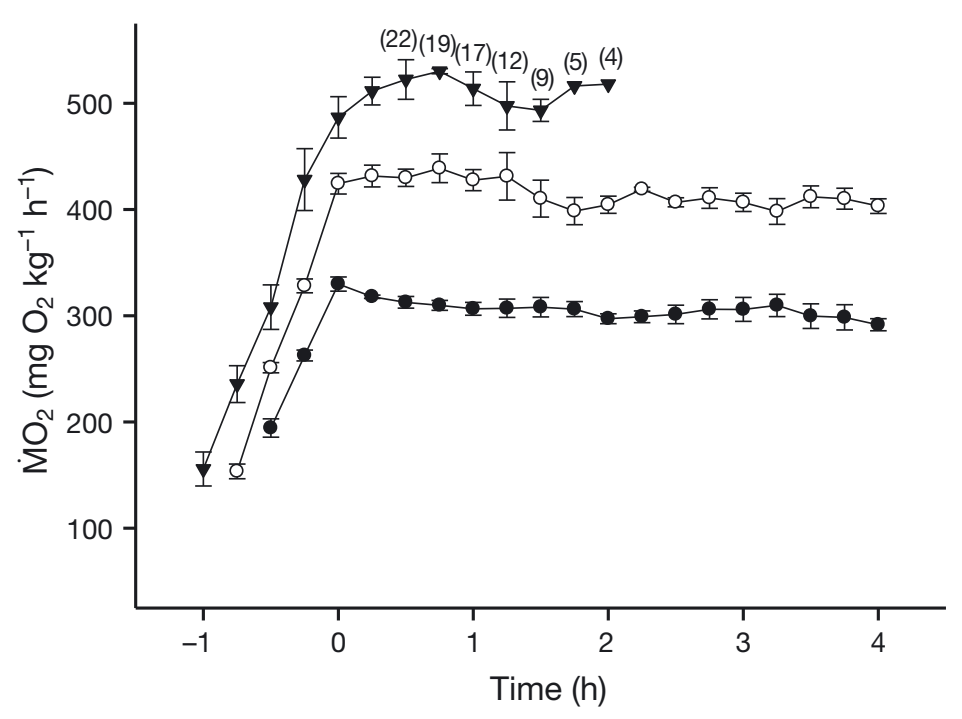

Fig. 2. Oxygen consumption rate $\left(\mathrm{MO}_{2}\right)$ during $4 \mathrm{~h}$ sustained swimming trials at $60 \%$ (closed circles), $80 \%$ (open circles) and $100 \%$ (triangles) of the average critical swimming speed $\left(U_{\text {crit }}\right)$ for this batch of Atlantic salmon corresponding to 58,78 and $97 \mathrm{~cm} \mathrm{~s}^{-1}$ respectively. Before $0 \mathrm{~h}, \mathrm{O}_{2}$ values correspond to the initial velocities prior to the desired swim speed being reached. Numbers in brackets: number of fish remaining; total number of fish at all other points is 24 . Data are means $\pm \mathrm{SE}$ 


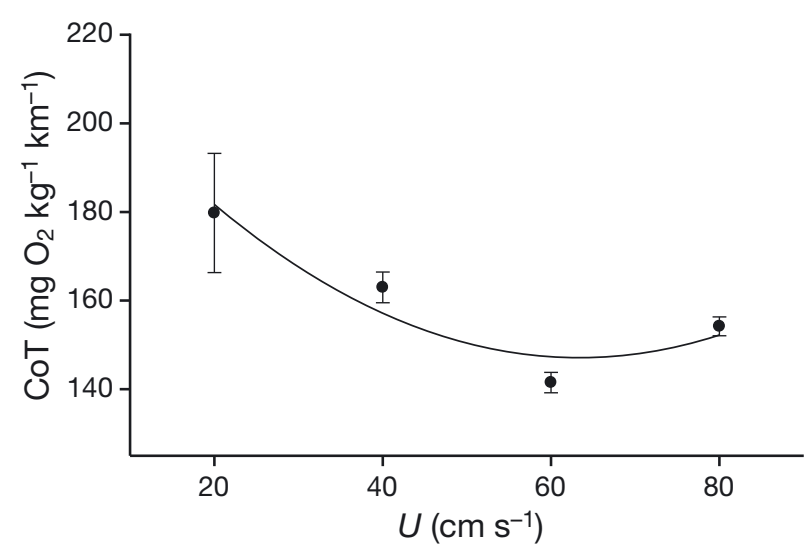

Fig. 3. Cost of transport (CoT) at intermediate swimming speeds calculated from the critical swimming speed $\left(U_{\text {crit }}\right)$ trials $(\mathrm{n}=3)$ for Atlantic salmon. The parabola fit is $y=$ $0.019 x^{2}-2.333 x+221, \mathrm{R}^{2}=0.903$, and the minimum is $147 \mathrm{mg} \mathrm{O}_{2} \mathrm{~kg}^{-1} \mathrm{~km}^{-1}$ at $63.3 \mathrm{~cm} \mathrm{~s}^{-1}$, which marks the theoretical optimum CoT. Data are means $\pm \mathrm{SE}$

The CoT plotted against what can be considered aerobic swimming speeds had a U-shaped curve, where the minimum of a parabola regression fit was $147 \mathrm{mg} \mathrm{O}_{2} \mathrm{~kg}^{-1} \mathrm{~km}^{-1}$ at $63.3 \mathrm{~cm} \mathrm{~s}^{-1}\left(1.47 \mathrm{BL} \mathrm{s}^{-1}\right)$ (Fig. 3). This minimum CoT represents a theoretical optimal cruising speed for long distance swimming.

Some variation in swimming performance was found, with $U_{\text {crit }}$ ranging from 1.84 to $2.79 \mathrm{BL} \mathrm{s}^{-1}$, and fatigue time at $100 \% U_{\text {crit }}$ ranging between 30 and 186 min (2 fish endured 240 min). Plots with RVM, $W$ and $K$ versus either relative $U_{\text {crit }}$ or fatigue time during sustained swimming can be seen in Fig. 4 with their corresponding Pearson's correlation coefficient and p-value. RVM and $K$ were not correlated with $U_{\text {crit }}(\mathrm{p}=$ 0.632 and 0.492 respectively), although $W$ was negatively correlated with the relative $U_{\text {crit }}(p=0.006)$. Fatigue time was independent of $W$ and $K(\mathrm{p}=0.606$ and 0.218 respectively), while fish with larger RVM were associated with earlier fatigue times $(\mathrm{p}=0.024)$.

\section{DISCUSSION}

\section{Sustained swimming capacity}

The average $U_{\text {crit }}$ of $97 \mathrm{~cm} \mathrm{~s}^{-1}\left(2.27 \mathrm{BL} \mathrm{s}^{-1}\right)$ found here is similar to earlier studies on reared Atlantic salmon when considering slight differences in fish size, temperature and experimental setups (Wagner et al. 2003, 2004, Wilson et al. 2007, Remen et al. 2016). In the 24 fish tested, $U_{\text {crit }}$ ranged from 80.5 to $107.3 \mathrm{~cm} \mathrm{~s}^{-1}$. This implies that during the sustained swimming trials at speeds derived from the group average $U_{\text {crit, }}$ individual fish experienced varying relative intensities centred on this group average. Using the minimum and maximum from the $U_{\text {crit }}$ trials, an estimate can be made for the range of individual variation in relative intensities experienced during the sustained trials: at $60 \%$ it would range from 54 to $72 \%$, at $80 \%$ from 73 to $97 \%$, and at $100 \%$ from 91 to $121 \%$. Therefore, some appreciable individual differences in experienced swimming intensity are likely to have occurred in the sustained swimming trials.

However, no fish became fatigued in either of the sub- $U_{\text {crit }}$ groups when forced to continue swimming for $4 \mathrm{~h}$, despite some individuals potentially swimming close to their $U_{\text {crit, }}$ particularly in the $80 \%$ group. This result is somewhat surprising considering a previous study on rainbow trout Oncorhynchus mykiss, which has respiratory physiology similar to Atlantic salmon, found significant intramuscular lactate production and phosphocreatine depletion within $30 \mathrm{~min}$ at 70 to $80 \% U_{\text {crit }}$ (Burgetz et al. 1998), while the aerobic limit for sustained swimming in salmonids is generally believed to be $\sim 80 \% U_{\text {crit }}$ (Webb 1971, Jones 1982). On the contrary, another study on Atlantic salmon found that white muscles were not recruited until speeds above $85.5 \% U_{\text {crit }}$ (Beddow \& McKinley 1999), which, together with the present study, suggest that Atlantic salmon possess a higher relative aerobic swimming capacity than other salmonids such as rainbow trout. Regardless, by testing sustained swimming capabilities for $\geq 200$ min according to the original definition by Beamish (1978) at a predefined sub-percentage of the average group $U_{\text {crit, }}$ it has now been confirmed that Atlantic salmon are able to sustain at least $80 \%$ of $U_{\text {crit }}$ for several hours.

\section{Oxygen consumption during sustained swimming}

$\mathrm{MO}_{2}$ while swimming at $80 \% U_{\text {crit }}$ for $4 \mathrm{~h}$ corresponded to $\sim 81.4 \%$ of the $\mathrm{VO}_{2 \max }$. It is therefore not surprising that no fish reached fatigue here, since the aerobic capacity was far from fully utilized at this swimming intensity.

Since intramuscular lactate accumulation was found at sub- $U_{\text {crit }}$ speeds in rainbow trout, and the fact that only 3 ATP molecules are produced when glycogen is converted to lactate while 5 ATP molecules are needed to convert lactate back into glycogen, it has been argued that anaerobic metabolism constitutes a notable component of the energy budget during salmonid migration (Burgetz et al. 1998). Furthermore, lactate recovery is slow in fish, ranging 

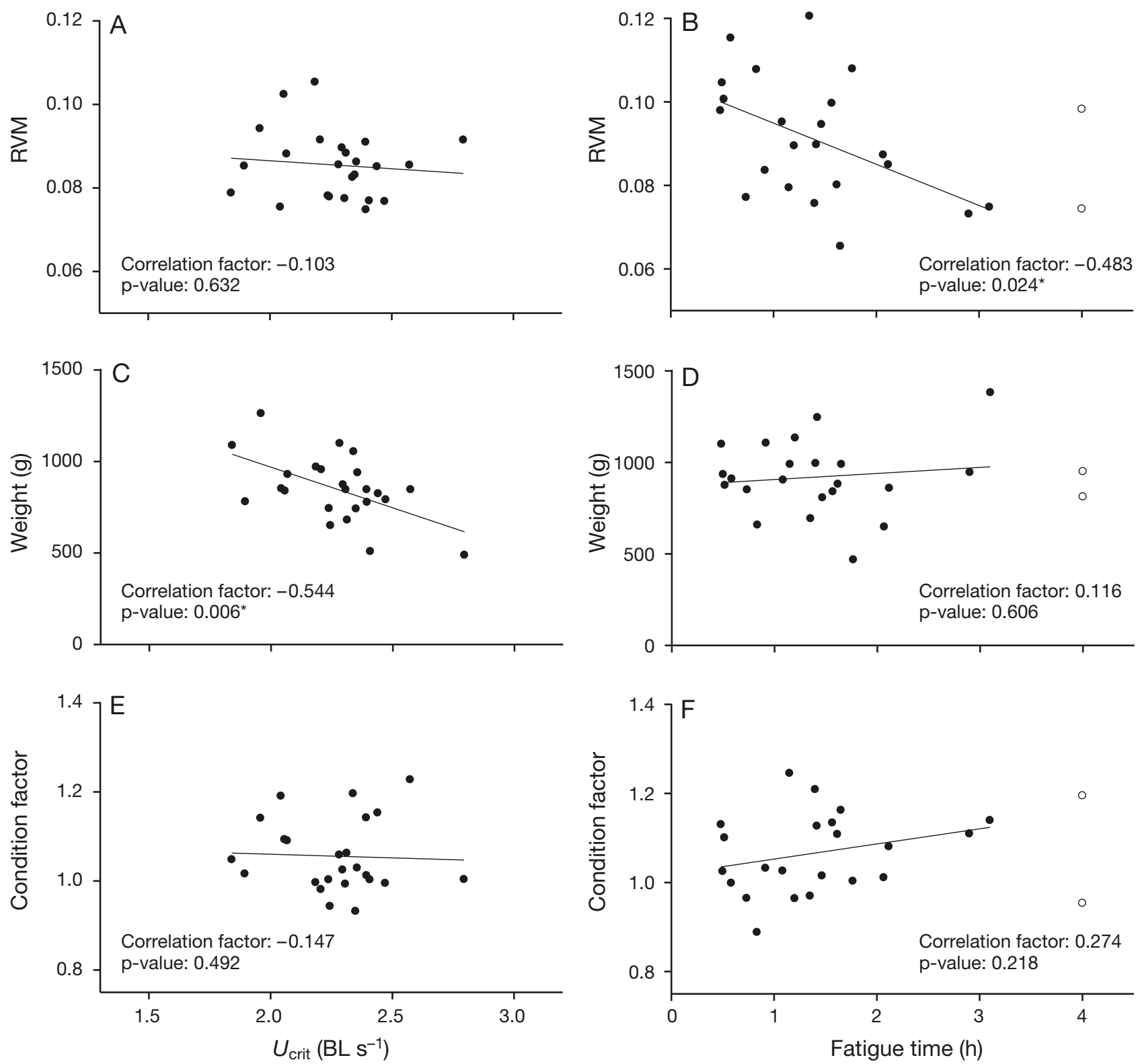

Fig. 4. Relative ventricular mass (RVM), weight, and condition factor versus either the relative critical swimming speed ( $\left.U_{\text {crit }}\right)$ (left panels) or fatigue time of Atlantic salmon during the sustained swimming challenge at $97 \mathrm{~cm} \mathrm{~s}^{-1}$ (right panels) $(\mathrm{n}=24$ ). Open circles on right panels: fish that completed the swimming trial and were therefore omitted from the correlation analyses. ${ }^{*} \mathrm{p}<0.05$

from 2 to $8 \mathrm{~h}$ (Schulte et al. 1992, Pagnotta et al. 1994). If swimming at sub- $U_{\text {crit }}$ required a substantial anaerobic component for Atlantic salmon, lactate would have to be metabolized 'on the run' to sustain homoeostasis, which should lead to an increase in $\mathrm{MO}_{2}$ over time when the fish were forced to swim at a high intensity for $4 \mathrm{~h}$. Theoretically, it could be assumed that during continued high intensity swimming an escalating excess post-exercise oxygen consumption (EPOC)-effect would eventually cause $\dot{\mathrm{MO}_{2}}$ to approach $\mathrm{VO}_{2 \text { max }}$ with a subsequent lactate accumulation and thereby cause fatigue. On the contrary, while swimming at 60 and $80 \% U_{\text {crit }}$ for $4 \mathrm{~h}$, $\dot{\mathrm{MO}_{2}}$ decreased significantly over time, meaning that the energy requirements of a potential anaerobic component were negligible in the energy budget for Atlantic salmon at these swimming intensities.

The observed decrease in $\dot{M O}_{2}$ over time may have been caused by more efficient swimming in the latter periods when the fish became more accustomed to the current velocity and optimised their swimming gait. Salmonids are dependent on anaerobic meta- 
bolism at the beginning of swimming and when swimming speeds increase, which is associated with lactate production and a small oxygen debt at intermediate velocities (Webb 1971, Jones 1982). Thus, some anaerobic metabolism may have occurred initially until a new steady-state rate of oxygen delivery to the working muscles was established. The decrease in $\mathrm{MO}_{2}$ over time could then, in part, also be ascribed to a decreasing need for lactate metabolism in the latter periods of the sustained swimming trials.

The minimum CoT can be interpreted as the optimum cruising speed for foraging or migration (Weihs 1973, Drenner et al. 2012). At $63.3 \mathrm{~cm} \mathrm{~s}^{-1}$ (1.47 BL $\mathrm{s}^{-1}$ ), the minimum CoT found in this study for Atlantic salmon corresponded to $65 \%$ of the $U_{\text {crit, }}$ and was far below the highest sustainable swimming speeds. This indicates that in theory, the optimum cruising speed should be achievable for an indefinite amount of time with no risk of fatigue. The minimum CoT reported here is similar to previous studies on salmonids (e.g. Lee et al. 2003, Hvas et al. 2017).

\section{Indicators of individual variation in swimming performance}

Differences in swimming capacity within groups of farmed Atlantic salmon may be related to differences in overall health and growth potential, and a thorough understanding of the magnitude and cause of this variation could be valuable in aquaculture practices. In a previous study, by screening the swimming capacity in a large group of Atlantic salmon parr and dividing them into 'good' and 'bad' swimmers, it was found that after smoltification, good swimmers had larger RVM, taller secondary lamellae, a thicker compact myocardial layer, higher maximum cardiac output and improved growth rates (Anttila et al. 2014).

In the present study, $U_{\text {crit }}$ ranged from 1.84 to $2.79 \mathrm{BL} \mathrm{s}^{-1}$ while time to fatigue at $97 \mathrm{~cm} \mathrm{~s}^{-1}$ ranged from $30 \mathrm{~min}$ to $\geq 4 \mathrm{~h}$ in samples of 24 larger postsmolts, which represents an appreciable variation in swimming capacity. However, RVM was not correlated with $U_{\text {crit, }}$ and surprisingly, RVM was negatively correlated with fatigue time in the sustained swimming trial. Although a crude indicator for cardiac function on its own, larger RVM are, however, associated with thicker compact myocardium which improves coronary circulation and assists in contractile power in Atlantic salmon (Anttila et al. 2014). Still, larger RVM was not associated with improved swimming performance in the present study. Too few fish may have been assessed to obtain robust pat- terns, or RVM may be less important in predicting swimming performance in larger Atlantic salmon.

Furthermore, $K$ and $W$ differences were also poor indicators of the observed variation in swimming performance within the size class tested here. Thus, the cause of variation in swimming performance is difficult to predict and is likely explained by numerous variables including cardio-respiratory traits. The relative role of various components in the swimming capacity variation in farmed Atlantic salmon deserves a more thorough and systematic assessment in future studies.

\section{Implications for exposed aquaculture}

The finding that farmed Atlantic salmon are able to sustain at least $80 \% U_{\text {crit }}$ for $4 \mathrm{~h}$ adds another important temporal dimension in the ongoing development of welfare guidelines for acceptable current velocities in exposed aquaculture. However, being forced to swim at intensities close to the sustainable limit chronically is not optimal in terms of production performance. When rainbow trout are swimming at $80 \%$ $U_{\text {crit, }}$ blood flow is redistributed in systemic circulation to favour working muscles while blood flow to the liver, spleen and stomach is decreased (Randall \& Daxboeck 1982). Continuous high-intensity aerobic swimming is therefore likely to inhibit digestion and growth (Farrell et al. 2001). This was recently demonstrated in Atlantic salmon post-smolts, where $6 \mathrm{wk}$ of forced swimming at $1.5 \mathrm{BL} \mathrm{s}^{-1}$ had a significantly negative impact on growth rate (Solstorm et al. 2015).

Furthermore, an important environmental factor to consider is low temperatures, since strong current events are more likely to occur during winter. In striped bass Morone saxatilis, carp Cyprinus carpio and rainbow trout, white muscles were recruited at lower swimming speeds at lower temperatures, which reduced the maximum sustainable swimming speed (Sisson \& Sidell 1987, Rome et al. 1990, Taylor et al. 1996). Similarly, $U_{\text {crit }}$ was significantly reduced at 3 and $8^{\circ} \mathrm{C}$ compared to $13^{\circ} \mathrm{C}$ in Atlantic salmon, where reduced tail beat frequencies and earlier transition to burst and glide swimming at lower temperatures suggested earlier white muscle recruitment (Hvas et al. 2017). Since the present study was conducted at $13^{\circ} \mathrm{C}$, the reported absolute values for sustainable swimming capacities are not representative of colder waters. Furthermore, it is unclear whether salmonids also are able to sustain $\sim 80 \% U_{\text {crit }}$ aerobically in suboptimal thermal environments. 
Finally, the original 200 min definition for sustainable swimming (Beamish 1978) may be inadequate for welfare assessment in salmonid aquaculture. The aetiology of fatigue can be either the inability to supply sufficient metabolites ( $U_{\text {crit }}$ protocols) or exhaustion of metabolite supply (fixed velocity tests) (Jones 1982). Like marathon runners, fish could therefore eventually 'hit the wall' due to substrate depletion, despite swimming within their aerobic limit. Current velocities that can be endured aerobically for 200 min may therefore still result in fatigue if exposures last for several days. Future studies should therefore seek to define swimming capabilities during periods of days or weeks at intermediate current exposure.

Acknowledgements. This study was funded by the Norwegian Research Council through the Centre for Researchbased Innovation in aquaculture technology, EXPOSED (237790). The authors declare no competing financial interests.

\section{LITERATURE CITED}

Anttila K, Jørgensen SM, Casselman MT, Timmerhaus G, Farrell AP, Takle H (2014) Association between swimming performance, cardiorespiratory morphometry, and thermal tolerance in Atlantic salmon (Salmo salar L.). Front Mar Sci 1:76

Beamish FWH (1978) Swimming capacity. In: Hoar WS, Randall DJ (eds) Fish physiology. Academic Press, New York, NY, p 101-187

Beddow TA, McKinley RS (1999) Importance of electrode positioning in biotelemetry studies estimating muscle activity in fish. J Fish Biol 54:819-831

Bell WH, Terhune LDB (1970) Water tunnel design for fisheries research. Technical Report No. 195, Fisheries Research Board of Canada, Nanaimo

Bjelland H, Føre M, Lader P, Kristiansen D and others (2015) Exposed aquaculture in Norway. In: Proc OCEANS' 15 MTS/IEEE, Washington, DC, p 19581967. http://ieeexplore.ieee.org/xpl/articleDetails.jsp? arnumber $=7404486$

Bone Q, Kicenuik J, Jones DR (1978) On the role of different fibre types in fish myotomes at intermediate swimming speeds. Fish Bull 76:691-699

Brett JR (1964) The respiratory metabolism and swimming performance of young sockeye salmon. J Fish Res Board Can 21:1183-1226

Brett JR (1967) Swimming performance of sockeye salmon in relation to fatigue time and temperature. J Fish Res Board Can 24:1731-1741

Bui S, Dempster T, Remen M, Oppedal F (2016) Effect of ectoparasite infestation density and life-history stages on the swimming performance of Atlantic salmon Salmo salar. Aquacult Environ Interact 8:387-395

Burgetz IJ, Rojas-Vargas A, Hinch SG, Randall DJ (1998) Initial recruitment of anaerobic metabolism during submaximal swimming in rainbow trout (Oncorhynchus mykiss). J Exp Biol 201:2711-2721

Cotterell SP, Wardle CS (2004) Endurance swimming of diploid and triploid Atlantic salmon. J Fish Biol 65:55-68

* Deslauriers D, Kieffer JK (2011) The influence of flume length and group size on swimming performance in shortnose sturgeon Acipenser brevirostrum. J Fish Biol 79:1146-1155

* Drenner SM, Clark TD, Whitney CK, Martins EG and others (2012) A synthesis of tagging studies examining the behaviour and survival of anadromous salmonids in marine environments. PLOS ONE 7:e31311

FFarrell AP (2007) Cardiorespiratory performance during prolonged swimming tests with salmonids: a perspective on temperature effects and potential analytical pitfalls. Philos Trans R Soc Lond B Biol Sci 362:2017-2030

Farrell AP, Thorarensen H, Axelsson M, Crockerd CE, Gamperl AK, Cech JJ (2001) Gut blood flow in fish during exercise and severe hypercapnia. Comp Biochem Physiol A 128:549-561

* Hartwell SI, Otto RG (1978) Swimming performance of juvenile menhaden (Brevoortia tyrannus). Trans Am Fish Soc 107:793-798

* Herskin J, Steffensen JF (1998) Energy savings in sea bass swimming in a school: measurements of tail beat frequency and oxygen consumption at different swimming speeds. J Fish Biol 53:366-376

* Holmer M (2010) Environmental issues of fish farming in offshore waters: perspectives, concerns and research needs. Aquacult Environ Interact 1:57-70

Hudson RCL (1973) On the function of the white muscles in teleosts at intermediate swimming speeds. J Exp Biol 58: 509-522

*Hvas M, Folkedal O, Imsland A, Oppedal F (2017) The effect of thermal acclimation on aerobic scope and critical swimming speed in Atlantic salmon Salmo salar. J Exp Biol 220:2757-2764

Jones DR (1982) Anaerobic exercise in teleost fish. Can J Zool 60:1131- 1134

Kiceniuk JW, Jones DR (1977) The oxygen transport system in trout (Salmo gairdneri) during sustained exercise. J Exp Biol 69:247-260

Lee CG, Farrell AP, Lotto A, MacNutt MJ, Hinch SG, Healy $\mathrm{MC}$ (2003) The effect of temperature on swimming performance and oxygen consumption in adult sockeye (Oncorhynchus nerka) and coho (O. kisutch) salmon stocks. J Exp Biol 206:3239-3251

* Pagnotta A, Brooks L, Milligan L (1994) The potential regulatory roles of cortisol in recovery from exhaustive exercise in rainbow trout. Can J Zool 72:2136-2146

Plaut I (2001) Critical swimming speed: its ecological relevance. Comp Biochem Physiol A Mol Integr Physiol 131: 41-50

* Pottinger TG, Carrick TR (1999) Modification of the plasma cortisol response to stress in rainbow trout by selective breeding. Gen Comp Endocrinol 116:122-132

Randall DJ, Daxboeck C (1982) Cardiovascular changes in the rainbow trout (Salmo gairdneri Richardson) during exercise. Can J Zool 60:1135-1140

* Remen M, Solstorm F, Bui S, Klebert P and others (2016) Critical swimming speed in groups of Atlantic salmon Salmo salar. Aquacult Environ Interact 8:659-664

Ricker WE (1975) Computation and interpretation of biological statistics of fish populations. Bull Fish Res Board Can 191:1-382

Rome LC, Funke RP, Alexander RL (1990) The influence of 
temperature on muscle velocity and sustained performance in swimming carp. J Exp Biol 154:163-178

Schulte PM, Moyes CD, Hochachka PW (1992) Integrating metabolic pathways in post-exercise recovery of white muscle. J Exp Biol 166:181-195

Sisson JE III, Sidell BD (1987) Effect of thermal acclimation on muscle fiber recruitment of swimming striped bass (Morone saxatilis). Physiol Zool 60:310-320

Solstorm F, Solstorm D, Oppedal F, Fernö A, Fraser TWK, Olsen RE (2015) Fast water currents reduce production performance of post-smolt Atlantic salmon Salmo salar. Aquacult Environ Interact 7:125-134

Svendsen JC, Skov J, Bildsoe M, Steffensen JF (2003) Intraschool positional preference and reduced tail beat frequency in trailing positions in schooling roach under experimental conditions. J Fish Biol 62:834-846

Taylor SE, Egginton S, Taylor EW (1996) Seasonal temperature acclimatisation of rainbow trout: cardiovascular and morphometric influences on maximal sustainable exercise level. J Exp Biol 199:835-845

Tudorache C, Viaenen P, Blust R, De Boeck G (2007) Longer flumes increase critical swimming speeds by increasing burst-glide swimming duration in carp Cyprinus carpio, L. J Fish Biol 71:1630-1638

Editorial responsibility: Chris Noble, Tromsø, Norway
Vindas MA, Johansen IB, Folkedal O, Höglund E and others (2016) Brain serotonergic activation in growth-stunted farmed salmon: adaptation versus pathology. R Soc Open Sci 3:160030

Wagner GN, McKinley RS, Bjørn PA, Finstad B (2003) Physiological impact of sea lice on swimming performance of Atlantic salmon. J Fish Biol 62:1000-1009

*Wagner GN, Balfry SK, Higgs DA, Lall SP, Farrell AP (2004) Dietary fatty acid composition affects the repeat swimming performance of Atlantic salmon in seawater. Comp Biochem Physiol A Mol Integr Physiol 137:567-576

* Webb PW (1971) The swimming energetics of trout II. Oxygen consumption and swimming efficiency. J Exp Biol 55:521-540

* Weihs D (1973) Optimal cruising speed for migrating fish. Nature 245:48-50

*Wilson RW, Egginton S (1994) Assessment of maximum sustainable swimming performance in rainbow trout (Oncorhynchus mykiss). J Exp Biol 192:299-305

* Wilson CM, Friesen EN, Higgs DA, Farrell AP (2007) The effect of dietary lipid and protein source on the swimming performance, recovery ability and oxygen consumption of Atlantic salmon (Salmo salar). Aquaculture 273:687-699

Submitted: February 15, 2017; Accepted: July 26, 2017

Proofs received from author(s): September 15, 2017 